\title{
Nicotinamide adenine dinucleotide phosphate oxidase inhibitor induces apoptosis on Epstein- Barr virus positive B lymphoma cells
}

\author{
Choong Heon $\mathrm{Ryu}^{1, \star}$, Sung Hyun Kim ${ }^{2, \star}$, Dae Young Hur ${ }^{3}$ \\ ${ }^{1}$ Department of Internal Medicine, Dongguk University Gyeongju Hospital, Gyeongju, ${ }^{2}$ Division of Pulmonary, Allergy and Critical Care Medicine, \\ Department of Internal Medicine, Inje University Busan Paik Hospital, Busan, ${ }^{3}$ Department of Anatomy and Tumor Immunology, Inje University \\ College of Medicine, Busan, Korea
}

\begin{abstract}
Over-expression of nicotinamide adenine dinucleotide phosphate oxidase (Nox) isoform enzymes was recently reported in various cancers including Burkitt's lymphoma (BL). However, the functions of Nox isoform enzymes in BL remain poorly understood. In this study, Nox isoform expression and the effects of a Nox-specific inhibitor were evaluated in Epstein-Barr virus (EBV)-positive Raji BL cells in comparison with EBV-negative Ramos BL cells. To evaluate Nox enzyme expression in Raji and Ramos BL cells, polymerase chain reaction (PCR) and western blot analysis were performed. To verify the intracellular signaling mechanism of the Nox inhibitor-induced apoptosis of Raji cells, WST-1 assay, trypan blue exclusion method, flow cytometry, PCR, western blotting, and bromodeoxyuridine staining were conducted. Experiments using the pan-caspase inhibitor $\mathrm{z}$-VAD, reactive oxygen species scavenger $\mathrm{N}$-acetyl-L-cysteine (NAC), and Bim inhibitor 1 were performed. PCR and western blot results showed that Nox isoform enzymes were highly expressed in EBV-positive BL Raji cells compared with EBV-negative BL Ramos cells. The Nox2 inhibitor induced apoptosis of Raji cells in time- and dosedependent manners. The Nox 2 inhibitor also caused up-regulation of Bim and Noxa, down-regulation of Mcl-1, translocation of Bax, release of cytochrome $c$, and caspase cascade activation, resulting in apoptosis. Furthermore, z-VAD, NAC, and BI-1 effectively blocked the Nox 2 inhibitor-induced apoptosis of Raji cells. Taken together, these results provide a novel insight into the mechanism of Nox inhibitor-induced apoptosis and evidence for Nox as a therapeutic target to treat EBV-positive malignancies.
\end{abstract}

Key words: Nicotinamide adenine dinucleotide phosphate oxidase, Burkitt lymphoma, Epstein-Barr virus, Apoptosis

Received October 23, 2020; Revised November 6, 2020; Accepted November 6, 2020

\section{Introduction}

Burkitt's lymphoma (BL) is a highly aggressive and inva-

Corresponding author:

Dae Young Hur (10)

Department of Anatomy and Tumor Immunology, Inje University

College of Medicine, Busan 47392, Korea

E-mail:dyhur@inje.ac.kr

*These two authors contributed equally to this work. sive B-cell non-Hodgkin lymphoma with varying patterns of clinical behavior [1]. BL patients are slowly decreasing and cure rates also improved. However, elder patients still have poor prognosis [2]. BL is associated with Epstein-Barr virus (EBV) infection and shows clinical symptoms that have been categorized into sporadic, endemic, and immunodeficiencyassociated subtypes. BL is characterized by translocations of chromosomes 8 and 14 that result in upregulation of the cmyc protein and subsequent increase of cell proliferation [3, 4]. With recent improvements in chemotherapy regimens and the application of new targeted therapies, the chance for

\section{Copyright ( 2020 . Anatomy \& Cell Biology}

This is an Open Access article distributed under the terms of the Creative Commons Attribution Non-Commercial License (http://creativecommons.org/licenses/by-nc/4.0/) which permits unrestricted non-commercial use, distribution, and reproduction in any medium, provided the original work is properly cited. 
successful treatment of children with BL has significantly improved [5, 6]. However, elderly patients and patients with advanced disease often show invasion to the central nervous system, and the prognosis for these patients is poor [7-9]. Therefore, the identification of an effective new treatment method for BL is urgently required. The development of a molecular-targeted therapy has presently taken center stage as the preferred treatment option.

Raji cells are derived from the B-lymphocytes of a male BL patient and categorized as lymphoblast-like. Some characteristics of Raji cells include a lack of differentiation, illustrated by the formation of large aggregations of hundreds of individual cells. Raji cells produce an unusual strain of EBV, which both transforms cord blood lymphocytes and induces early antigens in the cells $[10,11]$. Ramos cells originated from a human Caucasian BL male patient, and are EBV-genome-negative [12]. In the present study, these two cell lines were used as representative cell lines for BL.

EBV is a herpes virus type 4 which contains about 172 kilobase double strand DNA genome encoding about 100 products and non-coding RNAs. Latent viral proteins such as latent membrane proteins (LMPs) are associated with latent program in a host and deeply related to intracellular signal transduction, cellular regulation and cell proliferation of host cells as well as maintaining EBV genome [13].

Reactive oxygen species (ROS) plays an important role in various cell functions including the process of virus-induced malignant transformation $[14,15]$. A recent study reported that the increase in ROS level by EBV infection provides a growth advantage to EBV-infected cells by inducing the expression of cell proliferation-associated genes [15]. Regarding the role of ROS in B cell lymphoma including BL, another study reported that proliferation was induced through ROS increase [16]. Therefore, ROS is a potential therapeutic target in virus-associated cancers including BL. LMP1 regulates the expression Nox and Nox regulatory subunit p22phox [17].

The nicotinamide adenine dinucleotide phosphate (NADPH) oxidase (Nox) family is composed of the Nox1, $-2,-3,-4,-5$ and dual oxidase $1,-2$ proteins and includes the major enzymes responsible for the production of ROS [18, 19]. Over-expression of Nox isoform enzymes was recently reported in colorectal cancer and non-small cell lung cancer $[20,21]$. Furthermore, these enzymes play an important role in cell cycle regulation and have been proposed as a potential target in BL $[22,23]$. However, the effects of the specific inhibitors for Nox isoforms and the signaling mechanisms remain poorly understood.

The Bcl-2 family proteins are major regulators of apoptosis $[24,25]$. The pro-survival members (Bcl-2, Bcl-xL, Mcl-1, and $\mathrm{A} 1)$ are integral for cell survival; the $\mathrm{BH} 3$-only proteins (Bim, Puma, Bad, Noxa) initiate apoptosis signaling; and $\mathrm{Bax} / \mathrm{Bak}$ are required for mitochondrial outer membrane permeabilization [25-27]. The conformational changes of Bax and Bak lead to homo-oligomerization of the proteins and the formation of outer-mitochondrial membrane spanning pores [28]. These Bax and Bak pores permit the release of cytochrome $c$ (Cyt $c$ ) into the cytoplasm, which triggers activation of the caspase cascade, and ultimately results in apoptosis [29, 30].

In this study, we evaluated the expressions of Nox isoforms and the effects and cell signaling mechanism of a specific Nox inhibitor in EBV-positive Raji BL cells in comparison with EBV-negative Ramos BL cells.

\section{Materials and Methods}

\section{Cells and cell culture conditions}

Peripheral blood mononuclear cells (PBMCs) were isolated from whole blood by Ficoll-paque gradient centrifugation (Amersham Life Science, Buckinghamshire, UK). Primary B cells were purified from PBMCs using a magnetic-activated cell sorting B cell-negative depletion kit (Miltenyi Biotec, Bergisch Gladbach, Germany). Ramos, an EBV-negative B lymphoma cell line, and Raji, an EBV-positive B lymphoma cell line, were obtained from the American Type Culture Collection (Rockville, MD, USA). Both cell lines were maintained in RPMI-1640 medium (HyClone, Logan, UT, USA) containing $10 \%$ fetal bovine serum (HyClone) and antibiotics at $37^{\circ} \mathrm{C}$ in humiditied $5 \% \mathrm{CO}_{2}$ atmosphere.

\section{Reagents and antibodies}

Western blot was performed using the following primary antibodies: anti-human Nox2, anti-human Nox4 (BD Biosciences, San Diego, CA, USA), and anti-human $\beta$-actin antibodies (Santa Cruz Biotechnology, Santa Cruz, CA, USA). GSK2795039, a Nox2 inhibitor, was obtained from MedChemExpress (Monmouth Junction, NJ, USA). Anti-human caspase 3 and 9 antibodies (Abcam, Cambridge, MA, USA) and anti-human Mcl-1, Bcl-2, Bim, Noxa antibodies (Cell Signaling Technology, Beverly, MA, USA) were used to evaluate protein expression. N-Benzyloxycarbonyl-Val-Ala-Asp (OMe) fluoromethyl ketone (Z-VAD-FMK, Sigma, St. Louis, 
USA), a pan-caspase inhibitor; N-acetyl-L-cysteine (NAC, Cell Signaling Technology), a ROS scavenger; and ABT-263 (Selleck Chemical, Shanghai, China), a Bim specific inhibitor were used to identify the intra-cellular signaling pathway.

\section{Reverse-transcription polymerase chain reaction}

Cells were collected and washed three times with phosphate-buffered saline (PBS, $\mathrm{pH}=7.4$ ). Total RNA was isolated using the RNeasy Mini Kit (Qiagen, Hilden, Germany). RNA was transcribed into cDNA using the reverse-transcription PreMix Kit (Bioneer, Daejeon, Korea). Polymerase chain reaction (PCR) amplification was performed using primer sets (Bioneer) specific for Nox-2 (Forward: 5'-CAT GTT TCT GTA TCT CTG TGA-3'; Reverse: 5'-GTG AGG TAG ATG TTG TAG CT-3') and Nox-4 (Forward: 5'-CCA TGG CTG TGT CCT GGA GGA GCT G-3'; Reverse: 5'-AGT TGA GGG CAT TCA CCA GAT GGG C-3'). For the control, a specific primer set for $\beta$-actin (Forward: 5'-ATC CAC GAA ACT ACC TTC AA-3'; Reverse: 5'-ATC CAC ACG GAG TAC TTG C-3') was used, which yielded a 200-bp product. PCR products were visualized on $2.5 \%$ agarose gels with ethidium bromide. The normalization and fold-changes of levels of Nox 2 and Nox4 expression were calculated with Image J 1.38 software (http://rsweb.nih.gov/ij/index.html). Each experiment was repeated at least three times.

\section{Western blotting}

Cells were harvested and washed twice with PBS. Cells were lysed in radioimmunoprecipitation assay (RIPA) buffer (Elpis Biotech, Daejeon, Korea) containing a protease inhibitor cocktail (AEBSF, aprotinin, bestatin hydrochloride, E-64, EDTA, and leupeptin hemisulfate salt; Sigma). To evaluate phosphorylation events, an additional set of phosphatase inhibitors were added to the RIPA buffer (Cocktail II; sodium orthovanadate, sodium molybdate, sodium tartrate, and imidazole; Sigma). Protein concentration was estimated with the bicinochoninic acid Protein Assay Kit (Pierce, Rockford, IL, USA). Equal amounts of proteins $(40 \mu \mathrm{g})$ were separated by electrophoresis on sodium dodecyl sulfate-polyacrylamide gels and transferred to polyvinylidene difluoride membranes (Amersham) by immunoblotting. The membranes were then incubated overnight at $4^{\circ} \mathrm{C}$ in a PBS solution supplemented with $5 \%$ non-fat dry milk. The blots were probed with specific primary antibodies for 1 hour, incubated with diluted enzyme-linked secondary antibody, and then visualized by enhanced chemiluminescence as recommended by the man- ufacturer (Amersham). Equivalent protein loading was assessed by $\beta$-actin expression. Each experiment was repeated at least three times.

\section{Evaluation of cell viability}

Cells were seeded and then treated with GSK2795039 at the indicated doses for 48 hours, and cell viability was analyzed using a WST-1 assay (Sigma) or trypan blue exclusion method.

\section{Detection of apoptosis by Annexin V/propidium iodide}

Cells were harvested, washed twice with PBS and resuspended in $100 \mathrm{ml}$ Annexin V binding buffer $(10 \mathrm{mM}$ HEPES, $0.14 \mathrm{M} \mathrm{NaCl}$, and $0.25 \mathrm{mM} \mathrm{CaCl}_{2}$ ). Next, $2 \mathrm{ml}$ FITCconjugated Annexin V (BD Biosciences) and $1 \mathrm{ml}$ PI (BD Biosciences) were added, and cells were incubated at room temperature for 15 minutes in the dark with gentle vortexing. Next, $400 \mathrm{ml}$ of Annexin $\mathrm{V}$ binding buffer was added to each tube, and cells were analyzed using a flow cytometry.

\section{Measurement of cytochrome c and Bax translocation}

Mitochondrial and cytosolic fractions of cells were prepared using a Cytosol/Mitochondria fractionation kit (Calbiochem, San Diego, CA, USA). Briefly, cells were harvested by centrifugation at $600 \times \mathrm{g}$ for 5 minutes at $4^{\circ} \mathrm{C}$ and washed twice with cold PBS. Cells were then resuspended in 250 $\mathrm{ml}$ cytosol extraction buffer containing protease inhibitor mixture (included in the fractionation kit) and $1 \mathrm{mM}$ dithiothreitol (DTT). After incubation on ice for 10 minutes, cells were homogenized using a Dounce tissue homogenizer on ice. Homogenates were centrifuged at $700 \times \mathrm{g}$ for $10 \mathrm{~min}$ utes at $4^{\circ} \mathrm{C}$, and the supernatant was centrifuged again at $10,000 \times \mathrm{g}$ for 30 minutes at $4^{\circ} \mathrm{C}$. The resulting supernatants were harvested and designated as cytosolic fractions. The pellets were resuspended in $50 \mathrm{ml}$ mitochondria extraction buffer containing protease inhibitor mixture and DTT and designated as mitochondrial fractions. The fractionated cell lysates (10 mg per well) were prepared for western blotting using anti-Cox IV and anti-beta tubulin for loading control.

\section{Detection of sub-G level}

To detect the sub-Gı peak, PI staining was performed (BD Biosciences). Briefly, cells were incubated with Nox2 inhibitor, z-VAD, NAC, or Bim inhibitor in culture medium. Cells were collected and washed according to the instructions supplied by the manufacturer. Cell pellets were stained with PI 
staining solution containing RNase A (10 mg/ml; Sigma) and PI $(2 \mathrm{mg} / \mathrm{ml})$ in PBS. The cell suspension was then incubated in the dark at room temperature for 20 minutes. DNA content was determined using a FACSCalibur flow cytometer (BD Biosciences).

\section{Statistical analysis}

All data are expressed as mean \pm standard deviation and each value represents at least two different experiments. Comparisons between all individual data were made using one-way ANOVA. Statistical significance was defined by a $P$ value $<0.05$.

\section{Results}

\section{Nox2 and Nox4 were highly expressed in Raji cells compared with primary $B$ and Ramos cells}

Raji is an EBV-positive B lymphoma cell line, and Ramos is an Epstein-Barr-negative B lymphoma cell line. Recent
A
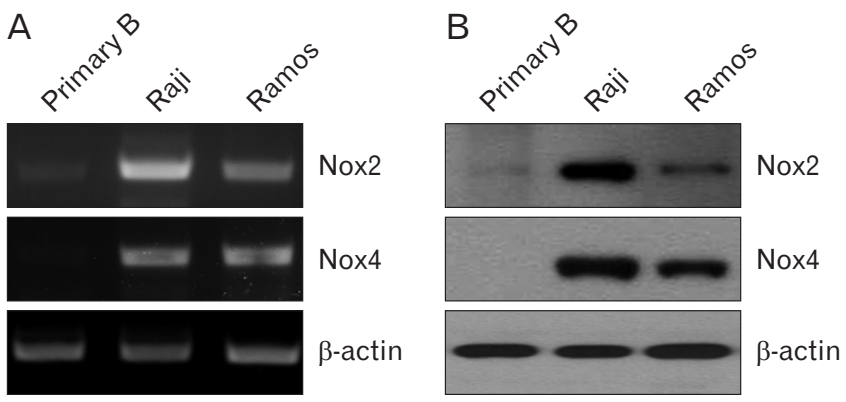

Fig. 1. PCR and western blot analyses of NADPH oxidase expression in primary B, Raji, and Ramos cells. (A) NADPH oxidase mRNA expression was verified by RT-PCR. $\beta$-actin was used as a loading control. Cells were harvested, and PCR amplification was performed as described as in the Materials and Methods. (B) Cells were harvested and cell lysates were extracted. The cell lysates were subjected to western blot using anti-human Nox $2,-4$, and $\beta$-actin antibodies. $\beta$-actin was used as a loading control. Results are representative of three independent experiments. NADPH, nicotinamide adenine dinucleotide phosphate; Nox2, NADPH oxidase 2; Nox4, NADPH oxidase 4; PCR, polymerase chain reaction; Primary B, primary B cells; RT, reverse-transcription.
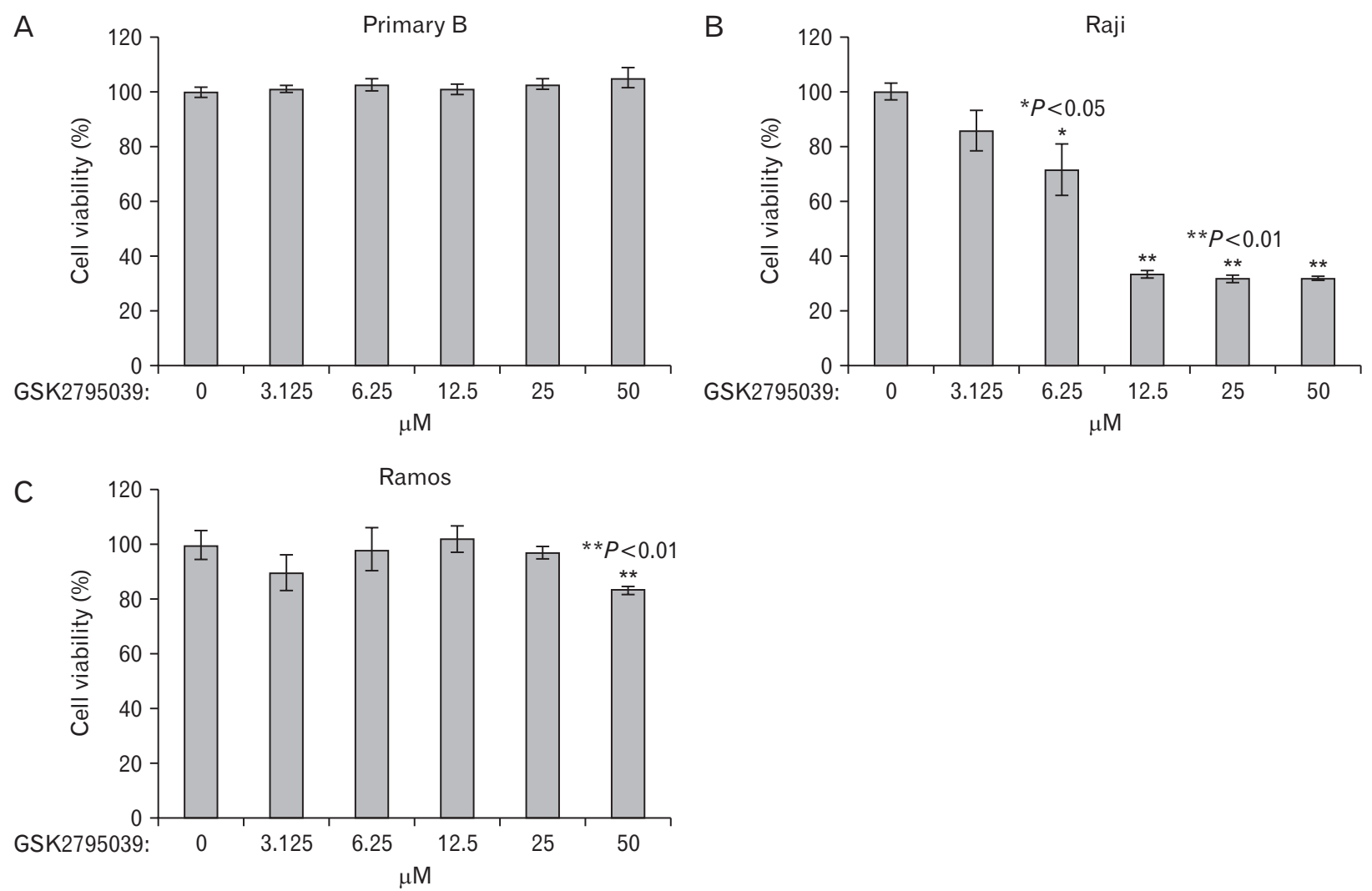

Fig. 2. Cell viability changes at 48 hours after Nox2 inhibitor, GSK2795039, treatment. Cells were seeded and treated with GSK2795039 at the indicated doses for 48 hours. Cell viability was analyzed using a WST-1 assay or trypan blue exclusion method. (A) Primary B cells, (B) Raji cells, (C) Ramos cells. Nox2, nicotinamide adenine dinucleotide phosphate oxidase 2; Primary B, primary B cells. The data shown are representative of three independent experiments $\left({ }^{*} P<0.05\right.$ vs. control, ${ }^{* *} P<0.01$ vs. control). 
studies reported that these two cell lines showed different characteristics including phenotypes [9-11]. Nox2 and Nox4 expression were first evaluated in primary B, Raji, and Ramous cells. PCR (Fig. 1A) and western blotting (Fig. 1B) results showed that Nox 2 mRNA and protein were highly expressed in Raji cells compared with the other cell lines. However, Nox4 protein only was highly expressed in Raji cells compared with the other cells.

\section{Nox2 inhibitor treatment induced cell death in Raji cells}

Our results showed that Raji cells significantly expressed Nox 2 mRNA and protein as evaluated by PCR and western blotting. To assess the role of Nox2, the cellular responses of Raji cells were observed after treatment using a specific inhibitor for Nox2, GSK2795039. The Nox 2 inhibitor significantly induced cell death in only Raji cells in a dose- dependent manner. The most effective concentration of GSK2795039 was $12.5 \mu \mathrm{M}$ concentration, and Raji cells showed the similar effects in higher concentration (Fig. 2B). GSK2795039 had no effect on primary B cells (Fig. 2A) and induced slight cell death in Ramos cells only at a high concentration; however, the induction of cell death was not statistically significant (Fig. 2C).

\section{Nox2 inhibitor induced apoptosis in Raji cells in time- and dose-dependent manners}

Our results showed that Nox2 inhibitor effectively induced cell death in only Raji cells. We next examined whether Nox2-induced cell death was associated with apoptosis. Cells treated with Nox2 inhibitor were stained with FITClabeled Annexin V and PI and analyzed by flow cytometry. Nox2 inhibitor treatment induced the apoptosis of Raji cells in a time-dependent manner (Fig. 3). Nox2 inhibitor treat-
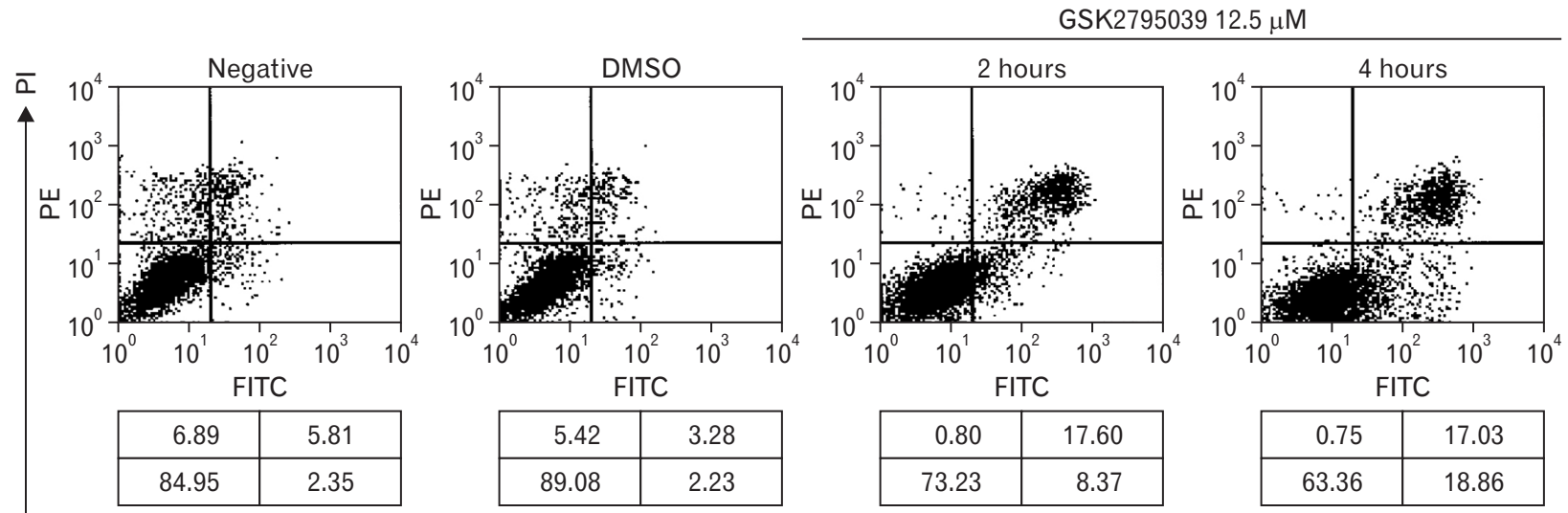

GSK2795039 $12.5 \mu \mathrm{M}$
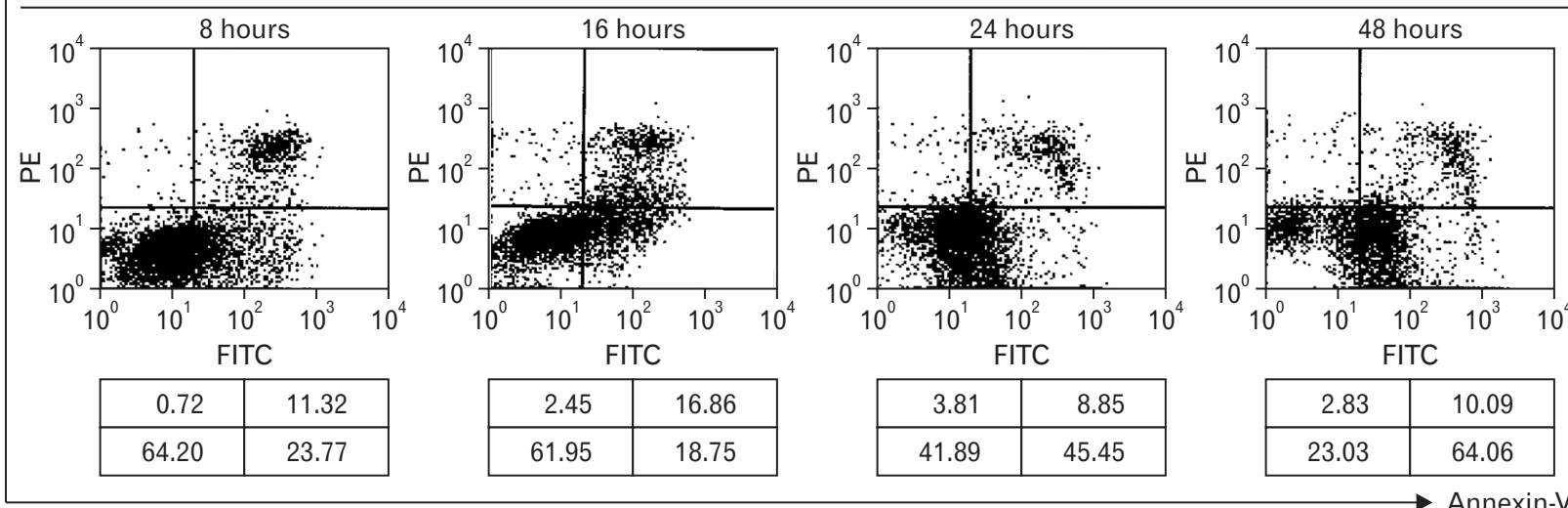

Fig. 3. Apoptosis analysis of Raji cells after Nox2 inhibitor, GSK2795039 treatment. Raji cells were treated with GSK2795039 for 48 hours. Cells were harvested, washed, and stained with FITC-conjugated Annexin V and PI as described in the Materials and Methods. Stained cells were analyzed by flow cytometry. The numbers in box represents the cell proportion in each quadrant. Data are representative of the three independent experiments. DMSO, control for GSK2795039; Negative, negative control; Nox2, nicotinamide adenine dinucleotide phosphate oxidase 2; PI, propidium iodide. 
A

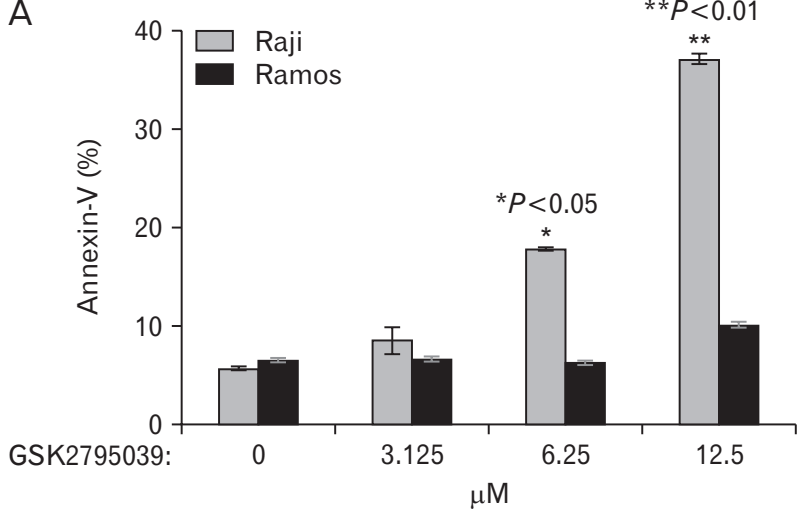

B

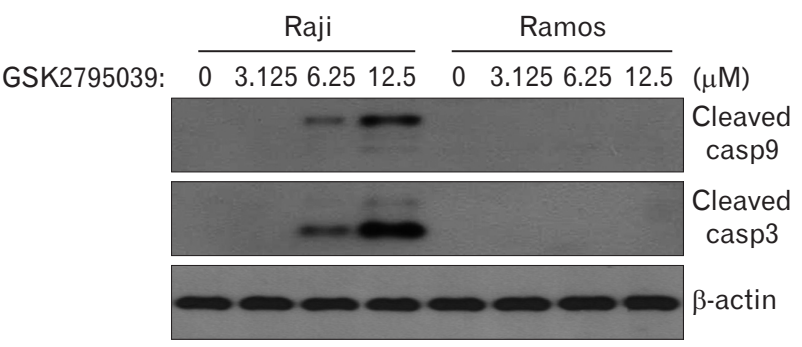

Fig. 4. GSK2795039, the Nox2 inhibitor, mediated caspase-dependent apoptotic cell death in Raji cells in a dose-dependent manner. (A) Raji and Ramos cells were treated with GSK2795039 in various concentrations for 48 hours and then stained with Annexin V-FITC. Apoptotic cell populations were determined with flow cytometry. ${ }^{*}$ and ${ }^{* *}$ indicate $P<0.05$ and $P<0.01$, respectively, compared with the Ramos cell apoptotic population. (B) Western blot analysis of caspase activation in cells after GSK2795039 treatment. $\beta$-actin was used as a loading control. Cell graphs represent the mean \pm standard deviation of three independent experiments in triplicate. Red box represents meaningful changes of molecule expressions. Nox2, nicotinamide adenine dinucleotide phosphate oxidase 2 .

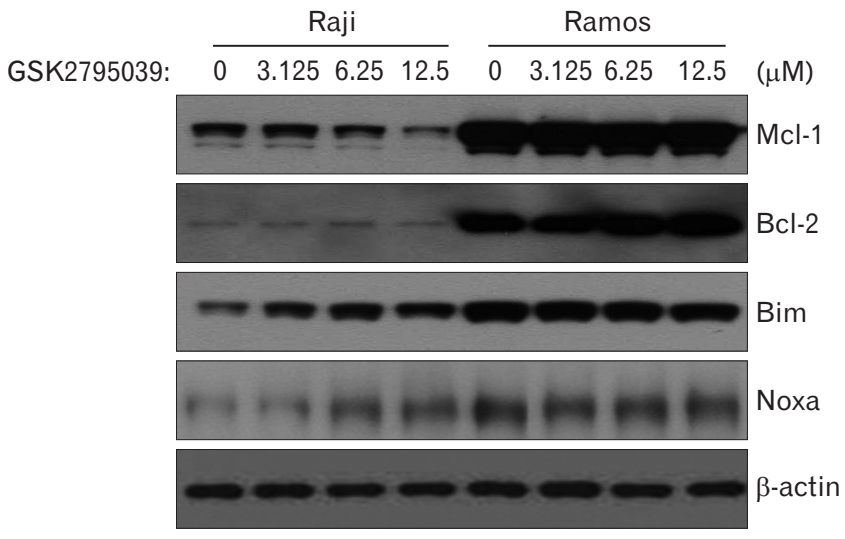

Fig. 5. Effects of the Nox inhibitor, GSK2795039, on Bcl-2 family proteins expressions in Raji and Ramos cells. After GSK2795039 treatment, cells were harvested and cell lysates were extracted. The cell lysates were subjected to western blot using anti-human Mcl-1, Bcl2 , Bim, Noxa, and $\beta$-actin antibodies. $\beta$-actin was used as a loading control. Results are representative of three independent experiments. Red box represents meaningful changes of molecule expressions.

ment also induced apoptotic cell death in Raji cells in a dosedependent manner, but no effects were observed in Ramos cells. The difference in Nox 2 inhibitor induced-apoptosis between Raji and Ramos cells were statistically significant at 6.25 and $12.5 \mu \mathrm{M}$ concentrations (Fig. 4A).

\section{Caspases are involved in Nox2 inhibitor-induced apoptosis}

Our results showed that the Nox2 inhibitor induced apoptosis in Raji cells. Caspases are key effector molecules that induce apoptosis in cancer cells [14]. Therefore, we next

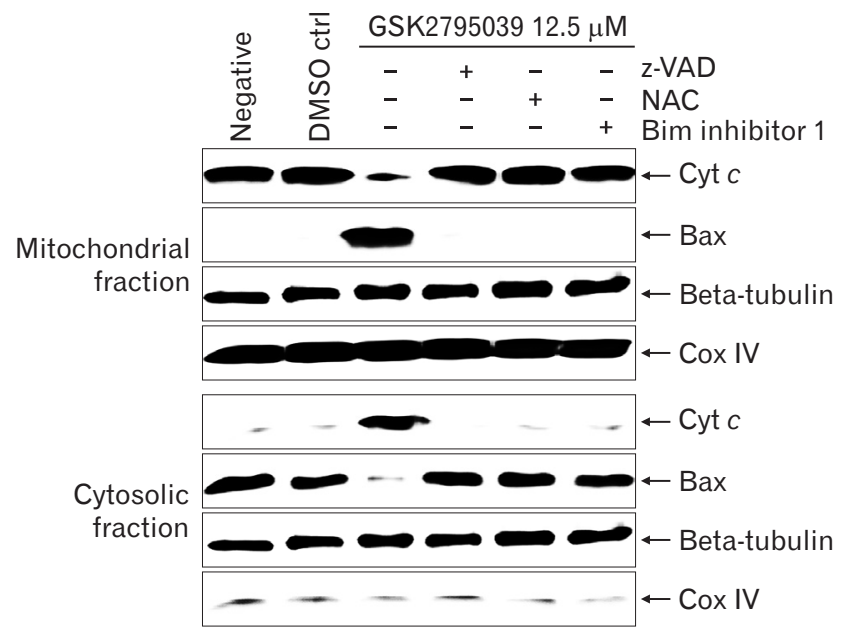

Fig. 6. Effects of GSK2795039 on the translocation of Bax and release of Cyt $c$ in Raji cells. After GSK2795039 treatment, cells were harvested and cell lysates were extracted. The cell lysates were separated into cytosolic and mitochondrial fractions, which were then analyzed by western blotting using anti-human Cyt $c$, bax, beta-tubulin, and Cox IV antibodies. Anti-Cox IV and anti-beta tubulin antibodies were used for fraction loading controls. Results are representative of three independent experiments. Cyt $c$, cytochrome c; DMSO ctr, solvent control; GSK2795039, Nox2 inhibitor; NAC, $\mathrm{N}$-acetyl-L-cysteine, reactive oxygen species scavenger; Negative, negative control without treatment; z-VAD, N-benzyloxycarbonylVal-Ala-Asp, a pan-caspase inhibitor.

evaluated the activation of caspases after Nox2 inhibitor treatment in Raji cells. The results showed that the Nox2 inhibitor induced the cleavage of caspases 3 and 9 (activated forms) in Raji cells in a dose-dependent manner, but no effects were observed in Ramos cells (Fig. 4B). 


\section{Mcl-1, Bim, and Noxa are involved in Nox2 inhibitor induced-apoptosis of Raji cells}

To identify the mechanism of Nox 2 inhibitor-induced apoptosis, several candidate signaling molecules were evaluated by western blot. Mcl-1, Bcl-2, Bim, and Noxa were expressed constitutively in both Raji and Ramos cells; GSK2795039 treatment decreased Mcl-1 expression and increased Bim and Noxa expression in Raji cells in a dosedependent manner, however, GSK2795039 had no effect on Ramos cells, similar to the previous results (Fig. 5).

\section{Translocation of Bax and release of cytochrome $c$ in Raji cells after GSK2795039 treatment}

Next, we examined translocation of Bax, a pro-apoptotic protein, from the cytoplasm to mitochondria and release of Cyt $c$ from the mitochondria into the cytoplasm after GSK2795039 treatment. Previous studies showed that the activation and translocation of Bim is associated with the translocation of Bax to mitochondria, and translocated Bax activates release of Cyt $c$, a key effector protein related with apoptosis [27, 28]. GSK2795039 treatment induced the translocation of Bax from the cytoplasm to mitochondria and release of Cyt $c$ from mitochondria into cytoplasm (Fig. 6).

\section{Inhibition assay using z-VAD, NAC, and BI-1 in Raji cells after GSK2795039 treatment}

To clarify the signaling pathway of Nox 2 inhibitor induced-apoptosis in Raji cells, inhibition assays were performed using the pan-caspase inhibitor z-VAD, the ROS scavenger NAC, and the Bim inhibitor $1 \mathrm{BI}-1 . \mathrm{z}-\mathrm{VAD}, \mathrm{NAC}$, and BI-1 effectively blocked the translocation of Bax and release of Cyt $c$ in Raji cells after GSK2795039 treatment (Fig. 6). Moreover, all three inhibitors completely restored the cell cycle arrest (subG1 phase in apoptotic cells) induced by GSK2795039 treatment (Fig. 7).
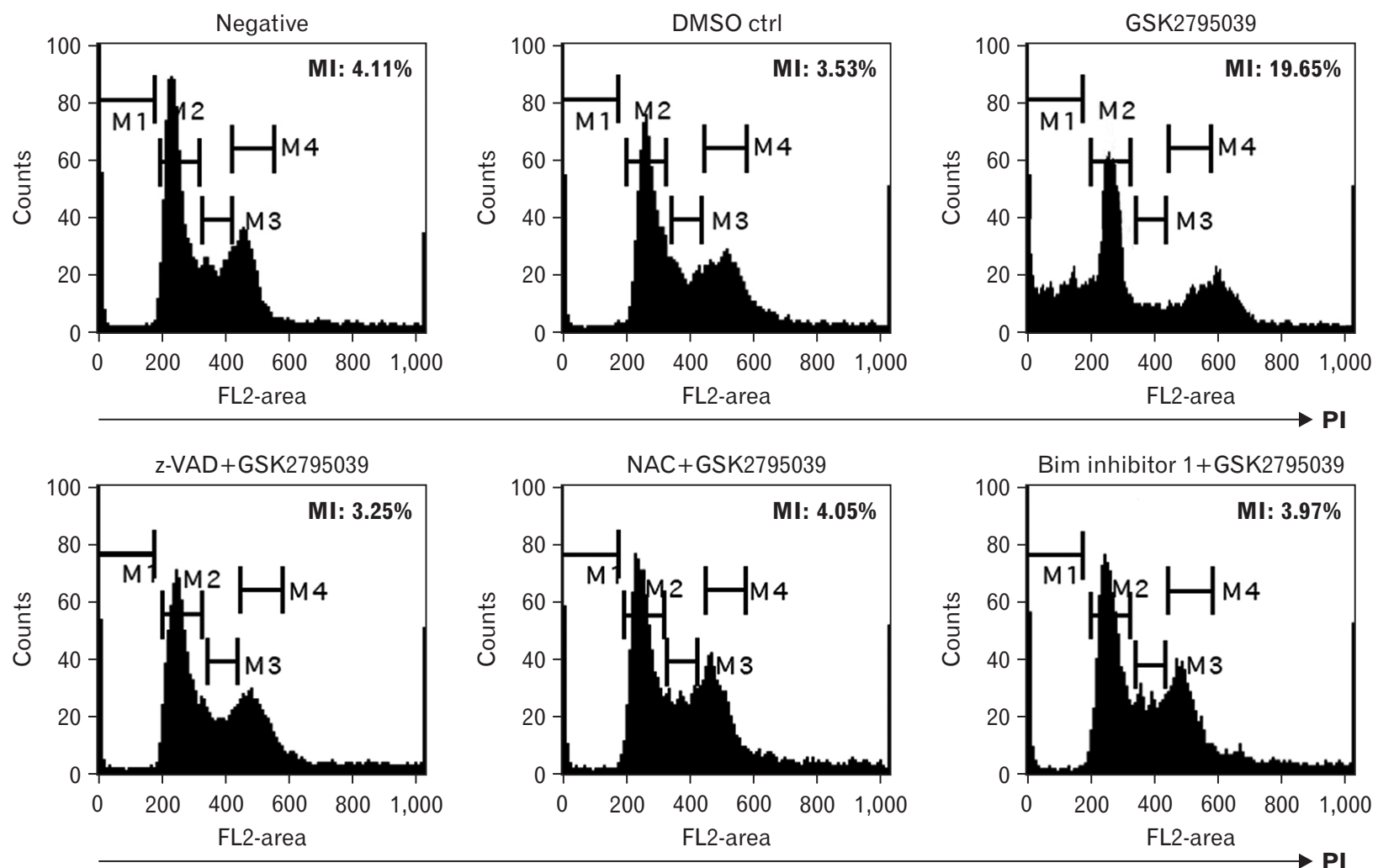

Fig. 7. Inhibitory effects of z-VAD, NAC, and Bim inhibitor 1 on the changes of sub-G $\mathrm{G}_{1}$ populations induced by GSK2795039 in Raji cells. To assess apoptotic cells, cells were stained with PI as described in the Materials and Methods. Cells were then analyzed by flow cytometry. The percentage represents the fraction of sub- $\mathrm{G}_{1}$ cells (M1) relative to the total number of cells. Results are representative of three independent experiments. DMSO ctr, solvent control; GSK2795039, Nox2 inhibitor; NAC, N-acetyl-L-cysteine, reactive oxygen species scavenger; Negative, negative control without treatment; z-VAD, N-benzyloxycarbonyl-Val-Ala-Asp, pan-caspase inhibitor. 


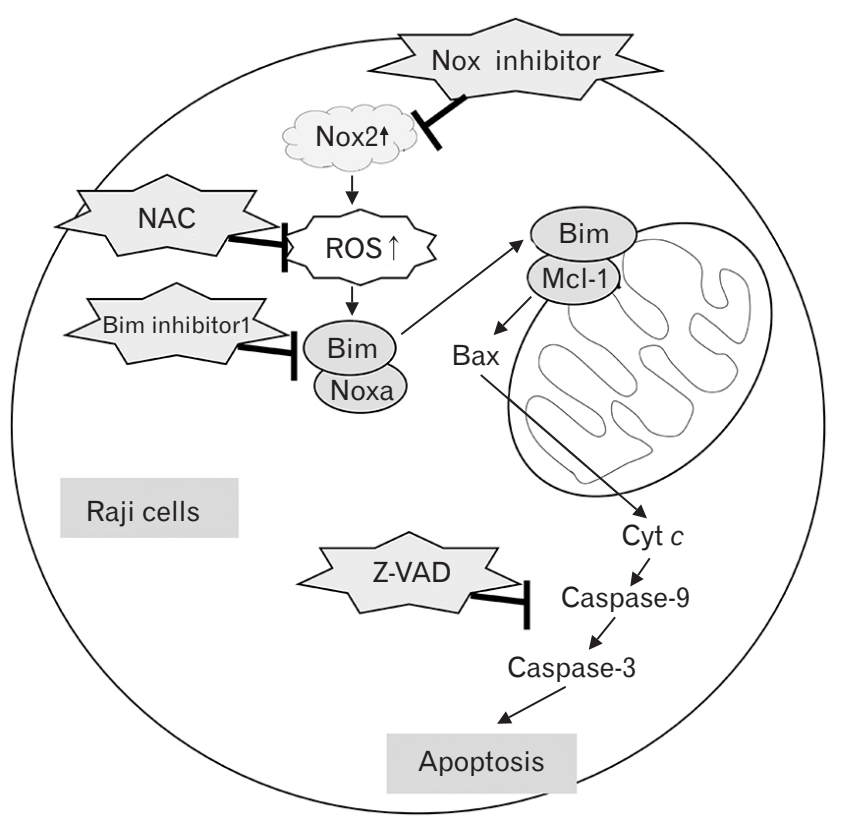

Fig. 8. Schematic diagram of the intracellular signaling mechanism during Nox 2 inhibitor-induced apoptosis in Raji cells. The increase of Nox2 expression effectively causes ROS generation as an initial response in EBV-positive B lymphoma Raji cells. Both the Nox inhibitor and ROS scavenger NAC effectively inhibit the cellular response following ROS generation. Aberrant ROS-mediated Bim and Noxa activation and Mcl-1 down-regulation induce the translocation of Bax to the mitochondria membrane, resulting in mitochondrial membrane potential disruption. The Bax pores cause the release of Cyt $c$ into the cytoplasm, resulting in caspase cascade activation and apoptosis. Cyt $c$, cytochrome $c$; EBV, Epstein-Barr virus; NAC, $\mathrm{N}$-acetyl-L-cysteine; Nox2, nicotinamide adenine dinucleotide phosphate oxidase 2; ROS, reactive oxygen species.

\section{Discussion}

Several previous studies have demonstrated that tumor virus infection is associated with increased oxidative stress through Nox induction, which exerts an important role in virus-induced transformation [29-31]. A recent study reported that EBV increases the proliferation of EBV-infected cells through the induction of ROS by cellular growth-associated factors in B lymphocytes [14].

In this study, the relationship between ROS-generating enzymes and EBV infection was assessed in two BL cell lines. We first investigated whether EBV infection could regulate the expression of the ROS-generating Nox enzyme family using EBV-positive Raji and EBV-negative Ramos BL cells. Our results showed that both Nox2 and Nox4 were highly expressed in EBV-positive Raji cells compared with EBVnegative Ramos cells. However, Nox 2 was more highly ex- pressed than Nox4 in Raji cells as shown by PCR and western blotting. Moreover, the Nox2 specific inhibitor GSK2795039 induced cell death only in Raji cells in a dose-dependent manner. The most effective concentration of GSK2795039 was $12.5 \mu \mathrm{M}$ concentration, therefore this concentration was used in the further experiments. These results suggested that Nox2 expression depends on EBV infection and could be a possible target for treatment in EBV-positive BL tumors. A recent study reported that the EBV oncoprotein LMP1 regulates the expression of Nox and a Nox regulatory subunit [32]. This report also supports the relationship between Nox enzymes and EBV infection.

We next evaluated whether apoptosis was involved in the cell death of Raji cells by GSK2795039 treatment using an Annexin-V/PI assay kit. GSK2795039 treatment induced the apoptosis of Raji cells in time- and dose-dependent manners, but no effect was observed in Ramos cells. In addition, GSK2795039 treatment induced the activation of caspase 3 and 9 in Raji cells in a dose-dependent manner. The pancaspase inhibitor z-VAD completely inhibited Nox inhibitorinduced apoptosis of Raji cells. These results indicated that GSK2795039 treatment induced the apoptosis of Raji cells in a caspase-dependent pathway.

Caspase activation is regulated at the multi-phase, including the release of other proteolytic enzymes such as Cyt $c$ from the mitochondria [33]. Moreover, Bcl-2 family proteins such as Bcl-2, Bcl-xL, Mcl-1, Bim, Puma, Noxa, Bad, and Bax regulate the release of apoptosis-associated proteins from mitochondria [34, 35]. Therefore, the signaling mechanism of Nox inhibitor-induced apoptosis was evaluated progressively using the verification of Bcl-2 family variation and inhibitor assay.

In this study, Nox2 inhibitor, GSK2795039, induced down-regulation of Mcl-1 and up-regulation of Bim and Noxa in a dose-dependent manner (as shown in Fig. 5). GSK2795039 induced the translocation of Bax and release of Cyt $c$. Previous studies reported that the translocation and conformational change of Bax leads to formation of outermitochondrial membrane spanning pores [26], and the Bax pores induce the release of Cyt $c$ into the cytoplasm, which triggers activation of the caspase cascade [27]. These previous studies were also consistent with the findings in this study. Based on the current results, we propose an intra-cellular signaling mechanism by the Nox2 inhibitor, GSK2795039, as summarized in Fig. 8. The increase of Nox2 expression effectively causes ROS generation as an initial response in Raji 
cells. Both the Nox inhibitor GSK2795039 and ROS scavenger NAC effectively inhibit the cellular response following ROS generation. Aberrant ROS-mediated Bim and Noxa activation and Mcl-1 down-regulation induce the translocation of Bax to the mitochondria membrane, resulting in mitochondrial membrane pores. These Bax pores cause the release of Cyt $c$ into the cytoplasm, inducing caspase cascade activation and apoptosis.

In summary, Nox enzymes were highly expressed in EBV-positive Raji cells. The Nox specific inhibitor induced up-regulation of Bim and Noxa, down-regulation of Mcl-1, translocation of Bax, release of Cyt $c$, and caspase cascade activation in Raji cells, resulting in apoptosis. The precise molecular mechanism and action sequence of the Nox inhibitor is not yet clear and should be examined in further studies.

\section{ORCID}

Choong Heon Ryu:

https://orcid.org/0000-0001-8455-7399

Sung Hyun Kim: https://orcid.org/0000-0002-5874-6767

Dae Young Hur: https://orcid.org/0000-0002-3964-7878

\section{Author Contributions}

Conceptualization: DYH. Data acquisition: CHR, SHK. Data analysis or interpretation: CHR, DYH. Drafting of the manuscript: CHR. Critical revision of the manuscript: DYH. Approval of the final version of the manuscript: all authors.

\section{Conflicts of Interest}

No potential conflict of interest relevant to this article was reported.

\section{Acknowledgements}

This work was supported by the 2019 Inje University research grant.

\section{References}

1. Kalisz K, Alessandrino F, Beck R, Smith D, Kikano E, Ramaiya $\mathrm{NH}$, Tirumani SH. An update on Burkitt lymphoma: a review of pathogenesis and multimodality imaging assessment of disease presentation, treatment response, and recurrence. Insights Imaging 2019;10:56.
2. Lu J, Tan H, Li B, Chen S, Xu L, Zou Y. Status and prognostic nomogram of patients with Burkitt lymphoma. Oncol Lett 2020;19:972-84.

3. Morton LM, Wang SS, Devesa SS, Hartge P, Weisenburger DD, Linet MS. Lymphoma incidence patterns by WHO subtype in the United States, 1992-2001. Blood 2006;107:265-76.

4. Ferry JA. Burkitt's lymphoma: clinicopathologic features and differential diagnosis. Oncologist 2006;11:375-83.

5. van Vuren A, Meyer-Wentrup F. New targets for antibody therapy of pediatric B cell lymphomas. Pediatr Blood Cancer 2014;61:2158-63.

6. Sandlund JT. Non-Hodgkin lymphoma in children. Curr Hematol Malig Rep 2015;10:237-43.

7. Dunleavy K. Approach to the diagnosis and treatment of adult Burkitt's lymphoma. J Oncol Pract 2018;14:665-71.

8. Casulo C, Friedberg J. Treating Burkitt lymphoma in adults. Curr Hematol Malig Rep 2015;10:266-71.

9. Molyneux EM, Rochford R, Griffin B, Newton R, Jackson G, Menon G, Harrison CJ, Israels T, Bailey S. Burkitt's lymphoma. Lancet 2012;379:1234-44.

10. Karpova MB, Schoumans J, Ernberg I, Henter JI, Nordenskjöld M, Fadeel B. Raji revisited: cytogenetics of the original Burkitt's lymphoma cell line. Leukemia 2005;19:159-61.

11. Drexler HG, Minowada J. History and classification of human leukemia-lymphoma cell lines. Leuk Lymphoma 1998;31:30516.

12. Spira G, Aman P, Koide N, Lundin G, Klein G, Hall K. Cellsurface immunoglobulin and insulin receptor expression in an EBV-negative lymphoma cell line and its EBV-converted sublines. J Immunol 1981;126:122-6.

13. Tao Q, Young LS, Woodman CB, Murray PG. Epstein-Barr virus (EBV) and its associated human cancers--genetics, epigenetics, pathobiology and novel therapeutics. Front Biosci 2006;11:2672-713.

14. Chen X, Kamranvar SA, Masucci MG. Oxidative stress enables Epstein-Barr virus-induced B-cell transformation by posttranscriptional regulation of viral and cellular growth-promoting factors. Oncogene 2016;35:3807-16.

15. Kgatle MM, Spearman CW, Kalla AA, Hairwadzi HN. DNA oncogenic virus-induced oxidative stress, genomic damage, and aberrant epigenetic alterations. Oxid Med Cell Longev 2017;2017:3179421.

16. Brandes RP, Weissmann N, Schröder K. Nox family NADPH oxidases: molecular mechanisms of activation. Free Radic Biol Med 2014;76:208-26.

17. Bonner MY, Arbiser JL. Targeting NADPH oxidases for the treatment of cancer and inflammation. Cell Mol Life Sci 2012;69:243542.

18. Cho SY, Kim JS, Eun HS, Kang SH, Lee ES, Kim SH, Sung JK, Lee BS, Jeong HY, Moon HS. Expression of Nox family genes and their clinical significance in colorectal cancer. Dig Dis Sci 2018;63:2332-40.

19. Leung EL, Fan XX, Wong MP, Jiang ZH, Liu ZQ, Yao XJ, Lu LL, Zhou YL, Yau LF, Tin VP, Liu L. Targeting tyrosine kinase 
inhibitor-resistant non-small cell lung cancer by inducing epidermal growth factor receptor degradation via methionine 790 oxidation. Antioxid Redox Signal 2016;24:263-79.

20. Ding Y, Zhu W, Sun R, Yuan G, Zhang D, Fan Y, Sun J. Diphenylene iodonium interferes with cell cycle progression and induces apoptosis by modulating $\mathrm{NAD}(\mathrm{P}) \mathrm{H}$ oxidase/ROS/cell cycle regulatory pathways in Burkitt's lymphoma cells. Oncol Rep 2015;33:1434-42.

21. Klingenberg M, Becker J, Eberth S, Kube D, Wilting J. The NADPH oxidase inhibitor imipramine-blue in the treatment of Burkitt lymphoma. Mol Cancer Ther 2014;13:833-41.

22. Suvarna V, Singh V, Murahari M. Current overview on the clinical update of Bcl-2 anti-apoptotic inhibitors for cancer therapy. Eur J Pharmacol 2019;862:172655.

23. Knight T, Luedtke D, Edwards H, Taub JW, Ge Y. A delicate balance- the BCL-2 family and its role in apoptosis, oncogenesis, and cancer therapeutics. Biochem Pharmacol 2019;162:25061.

24. Singh R, Letai A, Sarosiek K. Regulation of apoptosis in health and disease: the balancing act of BCL-2 family proteins. Nat Rev Mol Cell Biol 2019;20:175-93.

25. Huska JD, Lamb HM, Hardwick JM. Overview of BCL-2 family proteins and therapeutic potentials. Methods Mol Biol 2019;1877:1-21.

26. Peña-Blanco A, García-Sáez AJ. Bax, Bak and beyond- mitochondrial performance in apoptosis. FEBS J 2018;285:416-31.

27. Ugarte-Uribe B, García-Sáez AJ. Apoptotic foci at mitochon- dria: in and around Bax pores. Philos Trans R Soc Lond B Biol Sci 2017;372:20160217.

28. Luna-Vargas MPA, Chipuk JE. Physiological and pharmacological control of BAK, BAX, and beyond. Trends Cell Biol 2016;26:906-17.

29. Cerimele F, Battle T, Lynch R, Frank DA, Murad E, Cohen C, Macaron N, Sixbey J, Smith K, Watnick RS, Eliopoulos A, Shehata B, Arbiser JL. Reactive oxygen signaling and MAPK activation distinguish Epstein-Barr virus (EBV)-positive versus EBV-negative Burkitt's lymphoma. Proc Natl Acad Sci U S A 2005;102:175-9.

30. Williams VM, Filippova M, Filippov V, Payne KJ, DuerksenHughes P. Human papillomavirus type $16 \mathrm{E} 6^{*}$ induces oxidative stress and DNA damage. J Virol 2014;88:6751-61.

31. Fu N, Yao H, Nan Y, Qiao L. Role of oxidative stress in Hepatitis $\mathrm{C}$ virus induced hepatocellular carcinoma. Curr Cancer Drug Targets 2017;17:498-504.

32. Sun J, Hu C, Zhu Y, Sun R, Fang Y, Fan Y, Xu F. LMP1 increases expression of NADPH oxidase (Nox) and its regulatory subunit p22 in NP69 nasopharyngeal cells and makes them sensitive to a treatment by a Nox inhibitor. PLoS One 2015;10:e0134896.

33. Herr I, Debatin KM. Cellular stress response and apoptosis in cancer therapy. Blood 2001;98:2603-14.

34. Wang C, Youle RJ. The role of mitochondria in apoptosis*. Annu Rev Genet 2009;43:95-118.

35. Brenner D, Mak TW. Mitochondrial cell death effectors. Curr Opin Cell Biol 2009;21:871-7. 